\title{
Development of PPAR-agonist GW0742 as antidiabetic drug: study in animals
}

This article was published in the following Dove Press journal:

Drug Design, Development and Therapy

14 October 2015

Number of times this article has been viewed

\author{
Ho-Shan Niu' \\ Po-Ming $\mathrm{Ku}^{2,3}$ \\ Chiang-Shan Niu' \\ Juei-Tang Cheng ${ }^{3,4}$ \\ Kung-Shing $\mathrm{Lee}^{5-7}$ \\ 'Department of Nursing, Tzu Chi \\ College of Technology, Hualien \\ City, ${ }^{2}$ Department of Cardiology, \\ ${ }^{3}$ Department of Medical Research, \\ Chi-Mei Medical Center, Yong Kang, \\ Tainan City, ${ }^{4}$ Institute of Medical \\ Sciences, Chang Jung Christian \\ University, Guiren, Tainan City, \\ ${ }^{5}$ Department of Surgery, Division \\ of Neurosurgery, Pingtung Hospital, \\ ${ }^{6}$ Department of Surgery, Kaohsiung \\ Medical University, ${ }^{7}$ School of \\ Medicine, Chung-Ho Memorial \\ Hospital, Kaohsiung Medical \\ University, Kaohsiung City, Taiwan
}

Correspondence: Juei-Tang Cheng Institute of Medical Sciences, Chang Jung Christian University, Guiren, Tainan City 7II0I, Taiwan

Tel +886625 I 7864

Fax +88662832639

Email jtcheng@mail.cjcu.edu.tw

Kung-Shing Lee

Department of Surgery, Kaohsiung

Medical University, Kaohsiung City

8I20I, Taiwan

Tel +88673318839

Fax +88687365822

Email leekungshing@yahoo.com.tw
Background: The development of new drugs for the treatment of diabetes mellitus (DM) is critically important. Insulin resistance (IR) is one of the main problems associated with type-2 DM (T2DM) seen in clinics. GW0742, a selective peroxisome proliferator-activated receptor (PPAR)- $\delta$ agonist, has been shown to ameliorate metabolic abnormalities including IR in skeletal muscle in mice fed high-fructose corn syrup. However, the influence of GW0742 on systemic insulin sensitivity has still not been elucidated. Therefore, it is important to investigate the effect of GW0742 on systemic IR in diabetic rats for the development of new drugs.

Methods: The present study used a T2DM animal model to compare the effect of GW0742 on IR using homeostasis model assessment-IR (HOMA-IR) and hyperinsulinemic euglycemic clamping. Additionally, the insulinotropic action of GW0742 was investigated in type-1 DM (T1DM) rats. Changes in the protein expression of glucose transporter 4 (GLUT4) and phosphoenolpyruvate carboxykinase (PEPCK) in skeletal muscle and in liver, respectively, were also identified by Western blots.

Results: GW0742 attenuated the increased HOMA-IR in diabetic rats fed a fructose-rich diet. This action was blocked by GSK0660 at the dose sufficient to inhibit PPAR- $\delta$. Improvement of IR by GW0742 was also characterized in diabetic rats using hyperinsulinemic euglycemic clamping. Additionally, an increase of insulin sensitivity due to GW0742 was observed in these diabetic rats. Moreover, GW0742 reduced the hyperglycemia in T1DM rats lacking insulin. Western blotting analysis indicated that GW0742 reversed the decrease in GLUT4 and markedly reduced the increased PEPCK in liver.

Conclusion: The data showed that GW0742 has the ability to improve glucose homeostasis in diabetic rats through activation of PPAR- $\delta$. Therefore, PPAR- $\delta$ is a good target for the development of antidiabetic drugs in the future.

Keywords: insulin resistance, fructose-rich chow, HOMA-IR, streptozotocin, insulinotropic action, rats

\section{Introduction}

Diabetes mellitus (DM) is a disorder of metabolic homeostasis, showing hyperglycemia and altered lipid metabolism caused by dysfunction of pancreatic islets. ${ }^{1}$ The worldwide prevalence of DM among adults is predicted to increase from $6.4 \%$ to $7.7 \%$ ( 439 million) in 2030. ${ }^{2}$ Therefore, development of new drugs for the treatment of DM is important.

Clinically, type-2 DM (T2DM) is more prevalent than type-1 DM (T1DM). ${ }^{3}$ Insulin resistance (IR) is one of the major disorders in T2DM, with a marked reduction of insulin sensitivity. Induction of IR and development of T2DM-like animal models have been widely investigated in research by feeding high-fat diet ${ }^{4}$ or fructose-rich diet. ${ }^{5}$

Peroxisome proliferator-activated receptors (PPARs) heterodimerized with the retinoid X-receptor, a nuclear receptor, have been shown to be involved in lipid and 
carbohydrate metabolism after binding to peroxisome proliferator response element. ${ }^{6}$ All PPARs function mainly as transcription factors, ${ }^{7}$ and the PPAR family, including PPAR- $\alpha$, PPAR- $\gamma$, and PPAR- $\delta$, has widely been shown to exert regulatory roles in metabolic homeostasis. In particular, PPAR- $\delta$ is highly expressed in skeletal muscle, by approximately 10- and 50-fold more than PPAR- $\alpha$ and PPAR- $\gamma$, respectively, ${ }^{8}$ and is involved in obesity-associated IR. ${ }^{9}$ Many endogenous and synthetic ligands for PPARs have been identified. ${ }^{10}$ Recently, one study using knockout mice demonstrated that the improvement of glucose homeostasis by telmisartan, an angiotensin receptor blocker, involves selective PPAR- $\delta$ activation in skeletal muscle. ${ }^{11}$ Additionally, chronic administration of GW0742, a selective PPAR- $\delta$ agonist, in mice fed high-fructose corn syrup ameliorates the metabolic abnormalities, including IR, in skeletal muscle. ${ }^{12}$ However, the influence of GW0742 on systemic insulin sensitivity has still not been elucidated. Therefore, we investigated the effect of GW0742 on systemic IR in rats fed a fructose-rich diet.

In diabetes, elevation of blood glucose is a consequence of increased hepatic glucose output in concert with reduced peripheral glucose utilization, and phosphoenolpyruvate carboxykinase (PEPCK) is one of the key enzymes in hepatic gluconeogenesis. ${ }^{13}$ Additionally, insulin deficiency is clearly associated with a change in hepatic metabolism. ${ }^{13}$ Moreover, reduction in insulin-mediated glucose uptake caused by decreased expression of glucose transporter 4 (GLUT4) has been reported in diabetic skeletal muscle, a major site for glucose disposal. ${ }^{14}$ Therefore, we also focused on the changes in PEPCK and GLUT4 expression to evaluate the potential mechanism(s) for the glucose-lowering actions of GW0742.

\section{Materials and methods Experimental animals}

Male Wistar rats weighing 260-280 g were obtained from the Animal Center of the National Cheng Kung University Medical College. All rats were housed individually in plastic cages under standard laboratory conditions. The rats were maintained under a 12-hour light/dark cycle and had free access to food and water. All experiments were performed under anesthesia with sodium pentobarbital $(35 \mathrm{mg} / \mathrm{kg}$, intraperitoneal), and all efforts were made to minimize the animals' suffering. The animal experiments were approved and conducted in accordance with local institutional guidelines for the care and use of laboratory animals in Chi-Mei Medical Center, and the experiments conformed to the Guide for the Care and Use of Laboratory Animals, as well as the guidelines of the Animal Welfare Act.
Induction of T2DM by fructose-rich chow Rats were fed a 60\% fructose chow (Teklad Laboratory Diets, Madison, WI, USA), named fructose-rich chow, to induce a T2DM-like model showing IR as previously described. ${ }^{5}$ Tolbutamide (10 mg/kg, intraperitoneal)-induced hypoglycemia was used to characterize the success of induction in this model. The decrease of tolbutamide-induced action was observed in some rats after 4 weeks, and loss and/or marked reduction of responses occurred $\sim 8$ weeks later in all rats. We used these animals for further experiments in the present study.

\section{Induction of TIDM by injection of streptozotocin}

According to our previous report, ${ }^{15}$ overnight-fasted rats were intravenously injected with streptozotocin (STZ) $(60 \mathrm{mg} / \mathrm{kg})$ dissolved in $0.1 \mathrm{mmol} / \mathrm{L}$ citrate buffer ( $\mathrm{pH} 4.5)$. One week later, blood samples of each rat were used to assay the levels of glucose and insulin. Hyperglycemia and hypoinsulinemia were used to identify the success of this model, as described previously, ${ }^{15}$ and no mortality was observed during this induction.

\section{Drug treatment}

According to our previous method, ${ }^{16}$ the stock solutions of agonist GW0742 (Tocris Bioscience, Bristol, UK) or antagonist GSK0660 (Tocris Bioscience) were prepared in dimethyl sulfoxide. A fresh solution diluted with $9 \%$ normal saline to the indicated dose was used to treat the animals. To rule out the pharmacokinetic factors, GW0742 was intravenously injected at the indicated dose into animals under anesthesia. Additionally, GSK0660 was similarly injected 30 minutes before the injection of GW0742. The changes were then used to compare with the control, which was treated with the same volume of vehicle.

\section{Laboratory determinations}

The determination of plasma glucose was performed according to a previous study. ${ }^{15}$ Blood samples $(0.2 \mathrm{~mL})$ were collected from the femoral vein of rats under anesthesia with sodium pentobarbital (35 mg/kg, intraperitoneal). Blood samples were then centrifuged at 13,000 rpm for 3 minutes, and an aliquot $(15 \mu \mathrm{L})$ of plasma was added to $1.5 \mathrm{~mL}$ of glucose kit reagent (Biosystems SA, Barcelona, Spain) and incubated at $37^{\circ} \mathrm{C}$ for 10 minutes. The concentration of plasma glucose was measured in an analyzer (Quik-Lab, Ames, Spain; Miles Inc, Elkhart, IN, USA). The determination of plasma insulin was performed using a commercially available enzyme-linked immunosorbent assay (Linco, St Charles, MO, USA). 


\section{Identification of IR}

Development of IR in rats was characterized using the homeostasis model assessment-IR (HOMA-IR). Samples from each rat were analyzed in triplicate, and the results were expressed as microunits per milliliter of plasma insulin. HOMA-IR was then calculated according to the previous method $^{5}$ as follows: HOMA-IR $=$ fasting glucose $(\mathrm{mmol} / \mathrm{L}) \times$ fasting insulin $(\mu \mathrm{U} / \mathrm{mL}) / 22.5$.

\section{Hyperinsulinemic euglycemic clamping}

We performed hyperinsulinemic euglycemic clamping according to a previous report. ${ }^{16}$ After overnight fasting, rats under anesthesia with pentobarbital $(35 \mathrm{mg} / \mathrm{kg}$, intraperitoneal) received cannulation in the femoral vein for infusion of glucose and insulin, as well as receiving cannulation in the femoral artery for sampling. Before the experiment, animals were placed in a restrainer to become accustomed to the procedure. In the beginning, rats received infusions of regular human insulin (Novo Industrias, Bagsvaerd, Denmark) at $4 \mu \mathrm{U} / \mathrm{kg} / \mathrm{min}$. Insulin for infusion was diluted with saline containing 0.5\% human serum albumin (Baxter International Inc., Deerfield, IL, USA). The plasma glucose was measured immediately using the blood samples $(10 \mu \mathrm{L})$ collected at 10 -minute intervals. The plasma glucose level was then maintained at $5.5 \mathrm{mmol} / \mathrm{L}$ by infusion of $20 \%$ dextrose (Abbott Laboratories, Abbott Park, IL, USA). At the steady state generated within 70-90 minutes, the blood sample was collected to assay the glucose concentration. After the final sampling (120 minutes later), rats were sacrificed using a lethal dose of pentobarbital $(100 \mathrm{mg} / \mathrm{kg}$, intraperitoneal). We calculated the glucose infusion rate (GIR) at the steady state using Steele's equation, as described previously. ${ }^{16}$

\section{Analysis of insulin sensitivity}

After fasting overnight, all rats were challenged with exogenous insulin. Similar to our previous method, ${ }^{5}$ the insulin challenge test was performed by an intravenous injection of $0.5 \mathrm{IU} / \mathrm{kg}$ of short-acting human insulin into the rats. Blood samples $(0.2 \mathrm{~mL})$ from the femoral vein were drawn at the indicated time points following the intravenous insulin challenge test for the measurement of plasma glucose concentrations. Changes in the hypoglycemic response were used to identify insulin sensitivity.

\section{Western blot analysis}

The expression of metabolic regulatory signals in skeletal muscle or liver was examined using Western blot analysis as described in our previous report. ${ }^{16}$ In brief, ice-cold radioimmunoprecipitation assay buffer containing phosphatase and protease inhibitors $(50 \mathrm{mM}$ sodium vanadate, $0.5 \mathrm{mM}$ phenyl methyl sulfonyl fluoride, $2 \mathrm{mg} / \mathrm{mL}$ aprotinin, and $0.5 \mathrm{mg} / \mathrm{mL}$ leupeptin) was used for protein extraction. The protein concentrations were then measured by the bicinchoninic acid protein assay (Thermo Fisher Scientific, Waltham, MA, USA). Total protein samples ( $30 \mu \mathrm{g})$ were subjected to sodium dodecyl sulfate-polyacrylamide gel electrophoresis $(10 \%$ acrylamide gel) using the Bio-Rad Trans-Blot system and then transferred to membranes. The membranes were blocked with 5\% nonfat milk in Tris-buffered saline containing $0.1 \%$ Tween-20 (TBS-T), incubated for 60 minutes, and then hybridized with primary antibodies specific for PEPCK (Santa Cruz Biotechnology Inc., Dallas, TX, USA) or GLUT4 (Abcam, Cambridge, UK) at suitable concentrations in TBS-T for 16 hours. The membranes were then incubated with secondary antibody for an additional 3 hours. Incubation with secondary antibodies and detection of the antigen-antibody complex were performed using an ECL kit (Amersham Biosciences, Buckinghamshire, UK), and $\beta$-actin (Merck Millipore, Billerica, MA, USA) was used as the internal control. After comparing with the marker for specificity, the immunoblots of PEPCK (62 kDa), GLUT4 (54 kDa), and $\beta$-actin (43 kDa) were quantified with a laser densitometer.

\section{Statistical analysis}

Data are expressed as the mean \pm standard error of the mean. Statistical analysis was performed using one-way ANOVA analysis and Newman-Keuls post hoc analysis. $P$-value of $\leq 0.05$ was considered significant.

\section{Results}

\section{GW0742 decreases IR (HOMA-IR) in diabetic rats}

Rats became diabetic models after being fed fructose-rich chow for 2 months. Plasma levels of both glucose and insulin were markedly increased. Plasma glucose increased from $130.03 \pm 3.96 \mathrm{mg} / \mathrm{dL}(\mathrm{n}=8)$ to $135.36 \pm 2.64 \mathrm{mg} / \mathrm{dL} \quad(\mathrm{n}=8)$, and plasma insulin was increased from $30.84 \pm 1.4 \mu \mathrm{U} / \mathrm{mL}$ $(\mathrm{n}=8)$ to $74.89 \pm 4.74 \mu \mathrm{U} / \mathrm{mL}(\mathrm{n}=8)$. Therefore, the calculated HOMA-IR was also significantly $(P<0.05)$ increased from $9.91 \pm 0.62(\mathrm{n}=8)$ to $25.06 \pm 1.65(\mathrm{n}=8)$.

Treatment with GW0742 decreased the plasma glucose and insulin levels in the diabetic rats. As shown in Figure 1A, HOMA-IR was also reduced by GW0742 in a dose-related manner. Moreover, this action of GW0742 was reversed by pretreatment with the specific antagonist GSK0660 (Figure 1B). 

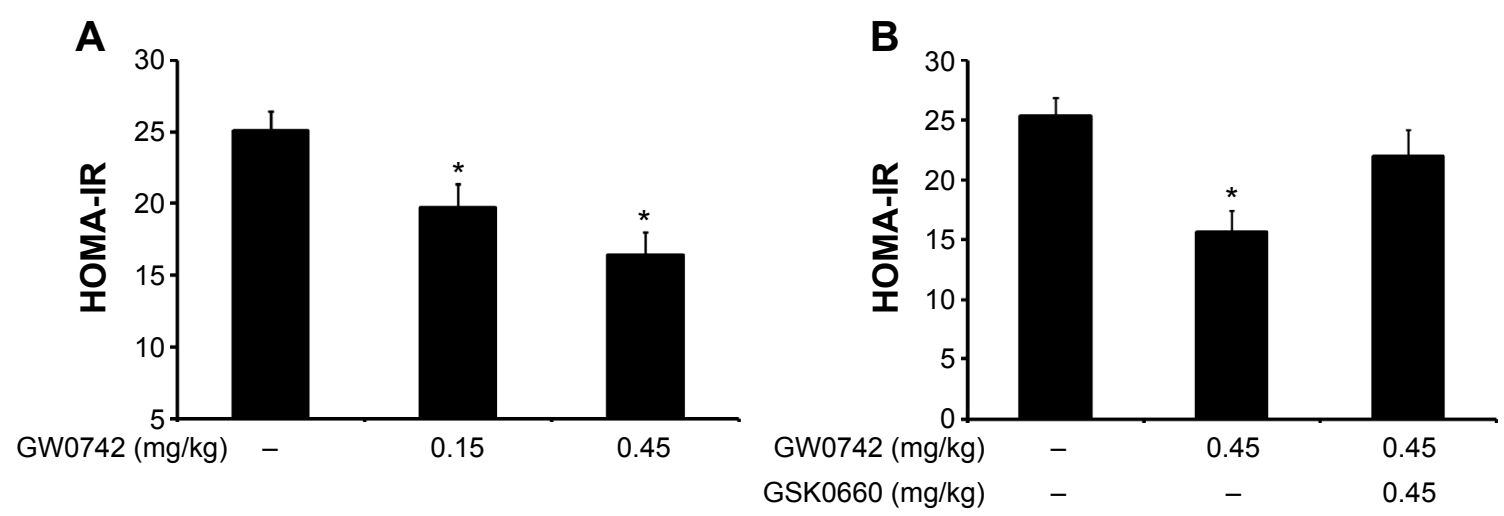

Figure I GW0742 changes HOMA-IR in diabetic rats.

Notes: (A) The dose-related inhibition of HOMA-IR induced by GW0742 in rats fed fructose-rich chow, and (B) the influence of PPAR- $\delta$ blockade induced by GSK0660. The HOMA-IR was calculated as described in the Methods section. Values (mean $\pm \mathrm{SE}$ ) were obtained from groups of eight animals each. $* P<0.05$ compared with vehicletreated group.

Abbreviations: GW0742, selective PPAR- $\delta$ agonist; HOMA-IR, homeostasis model assessment-insulin resistance; PPAR, peroxisome proliferator-activated receptor; SE, standard error.

\section{Hyperinsulinemic euglycemic clamping shows improvement of IR by treatment with GW0742 in diabetic rats}

The GIR obtained using the hyperinsulinemic euglycemic clamp is widely applied to identify IR. ${ }^{17}$ As shown in Figure 2, the GIR was markedly decreased in diabetic rats compared to that in normal rats. GW0742 reversed this decrease of GIR in a dose-related manner (Figure 2). Thus, these data showed that GW0742 improves IR.

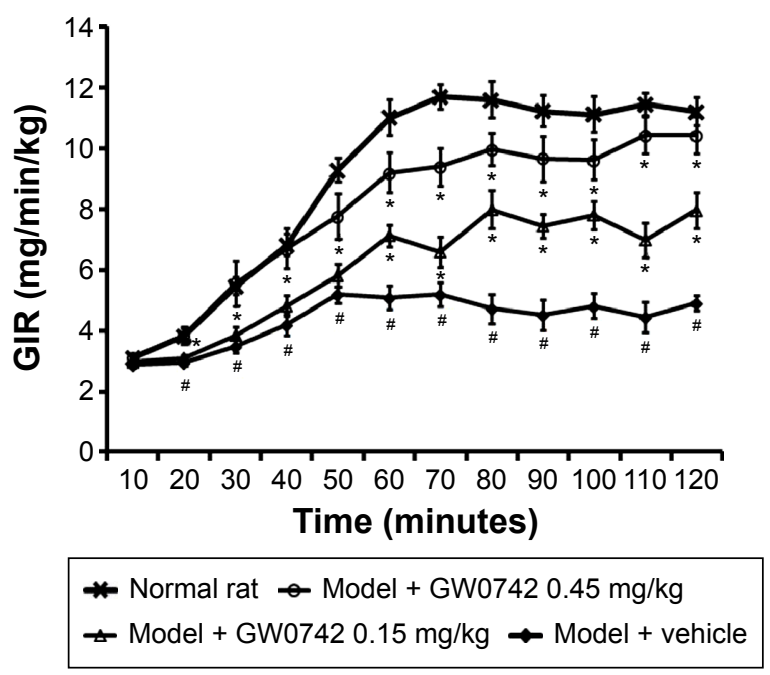

Figure 2 Changes in the GIR induced by GW0742.

Notes: The data from the diabetes model induced by fructose-rich chow are shown as Model, and data from the treatment of GW0742 are shown as Model + GW0742 at the indicated dose. Additionally, changes in normal rats were used for comparison with the vehicle-treated diabetic rats (Model + vehicle). The GIR was calculated as described in the Methods section. Values (mean $\pm \mathrm{SE}$ ) were obtained from groups of eight animals each. $* P<0.05$ compared with Model + vehicle and ${ }^{\# P}<0.05$ compared with the vehicle-treated normal rats (Normal rat).

Abbreviations: GIR, glucose-infusion rate; GW0742, selective PPAR- $\delta$ agonist; PPAR, peroxisome proliferator-activated receptor; SE, standard error.

\section{GW0742 enhanced insulin sensitivity in diabetic rats}

For understanding the change in insulin sensitivity, we investigated the responses to exogenous insulin as previously described. ${ }^{5}$ As shown in Figure 3, the hypoglycemic activity of insulin in diabetic rats was also enhanced by GW0742 in a dose-related manner.

\section{Direct effect of GW0742 on glucose homeostasis in type I-like diabetic rats}

To understand the direct effect of GW0742 on glucose utilization, we used STZ-induced type 1-like diabetic rats, as

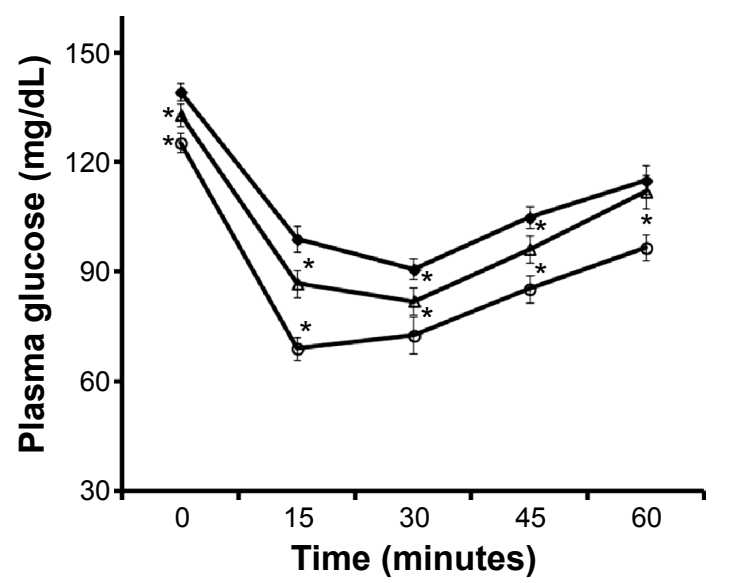

Vehicle ¿ GW0742 $0.15 \mathrm{mg} / \mathrm{kg}$ - GW0742 $0.45 \mathrm{mg} / \mathrm{kg}$

Figure 3 Changes in insulin sensitivity induced by GW0742 in diabetic rats. Notes: Data from the diabetes model receiving vehicle treatment are shown as Vehicle, and data from the GW0742-treated diabetic rats are shown as GW0742 at the indicated dose. The insulin sensitivity was assessed as described in the Methods section. Values (mean $\pm \mathrm{SE}$ ) were obtained from groups of eight animals each. $* P<0.05$ compared with the vehicle-treated diabetic rats.

Abbreviations: GW0742, selective PPAR- $\delta$ agonist; PPAR, peroxisome proliferatoractivated receptor; SE, standard error. 
A

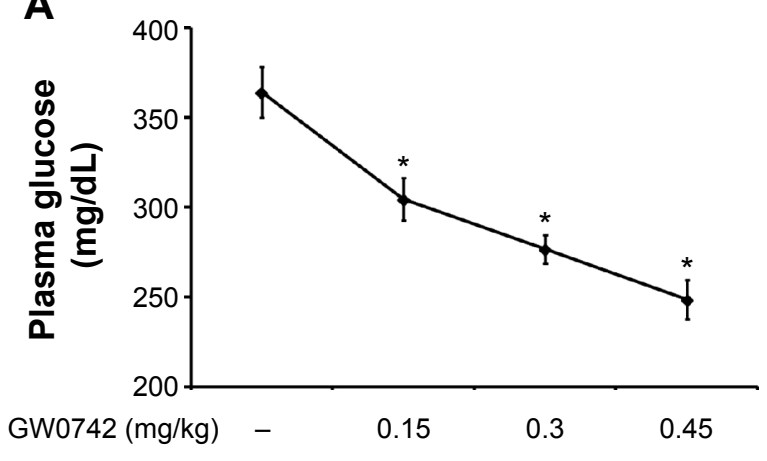

B

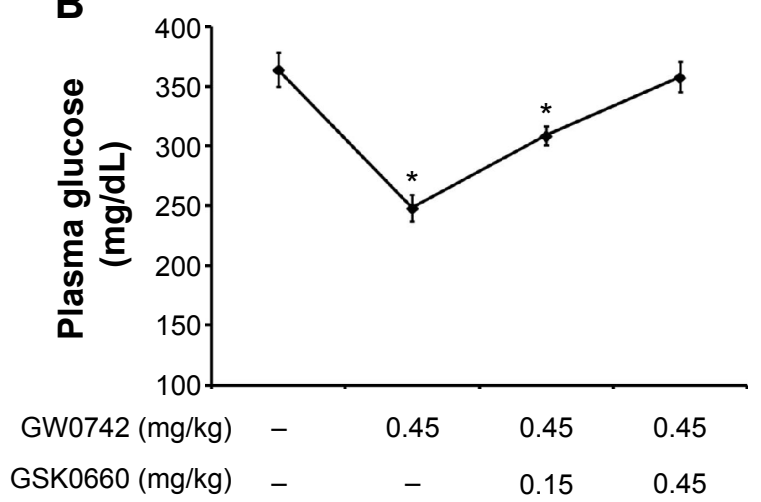

Figure 4 Effect of GW0742 on the plasma glucose levels in type I-like diabetic rats.

Notes: (A) Changes of plasma glucose induced by GW0742 in streptozotocin-induced diabetic rats, and (B) the influence of PPAR- $\delta$ blockade by treatment with GSK0660. The plasma glucose was measured as described in the Methods section. Values (mean $\pm \mathrm{SE}$ ) were obtained from groups of eight animals each. $* P<0.05$ compared with the vehicle-treated diabetic rats.

Abbreviations: GW0742, selective PPAR- $\delta$ agonist; PPAR, peroxisome proliferator-activated receptor; SE, standard error.

described in our previous report. ${ }^{18}$ Two weeks after injection of STZ, the rats became type 1-like diabetic, showing plasma glucose levels $\geq 300 \mathrm{mg} / \mathrm{dL}$. Additionally, the plasma insulin in STZ-induced diabetic rats was only $4 \%$ or less of that in normal rats. Therefore, mediation of endogenous insulin is negligible in this type 1-like diabetic model as described previously. ${ }^{18}$

GW0742 decreased the hyperglycemia in diabetic rats lacking insulin in a dose-dependent manner, as shown in Figure 4A. This inhibition was also reversed by blockade of PPAR- $\delta$ using GSK0660 (Figure 4B). Therefore, these data indicated a direct effect of PPAR- $\delta$ activation on glucose homeostasis.

Following our previous method, ${ }^{18}$ protein levels of GLUT4 in skeletal muscle and PEPCK in the liver of these diabetic rats were determined. After daily treatment of GW0742 at an effective dose for 1 week, the liver and soleus muscle were removed, frozen in liquid nitrogen, and stored at $-70^{\circ} \mathrm{C}$ until determination by Western blotting analysis. As shown in Figure 5, the decreased GLUT4 expression in the soleus muscle of diabetic rats was reversed by GW0742 in a dose-related manner. Additionally, the increased hepatic PEPCK level in diabetic rats was also markedly reduced by GW0742 in a same manner (Figure 6).

\section{Discussion}

The present study showed that the IR induced in rats receiving fructose-rich chow was improved by GW0742 through activation of PPAR- $\delta$. Insulinotropic action of GW0742 was also identified in diabetic rats lacking insulin. Therefore, these data suggest that activation of PPAR- $\delta$ by GW0742 may help the treatment of diabetic disorders in animals.
GW0742 is known to be an activator of PPAR- $\delta,{ }^{19}$ and it can increase the expression of PPAR- $\delta .{ }^{20}$ Therefore, GW0742 is widely used as an agonist of PPAR- $\delta$ in research. This study showed that GW0742 reduces HOMA-IR in diabetic rats fed a fructose-rich diet. This action was markedly reversed by GSK0660 at the dose sufficient to block PPAR- $\delta .{ }^{21}$ Thus, these data indicate that activation of PPAR- $\delta$ improves systemic IR. Additionally, a hyperinsulinemic euglycemic clamp was applied to assay the IR because this is the most reliable method

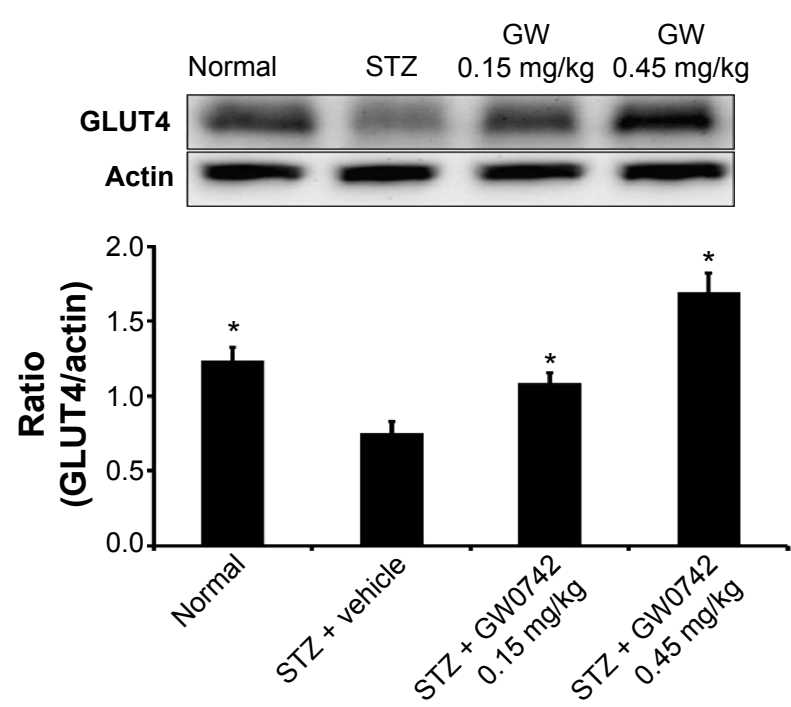

Figure 5 Changes of GLUT4 expression induced by GW0742 in the soleus muscle of type I-like diabetic rats.

Notes: Changes of GLUT4 expression in STZ-induced diabetic rats are shown as STZ for comparison with the changes in normal rats, Normal. Additionally, effects of GW0742 on diabetic rats are shown as STZ + GW0742 at the indicated dose compared to diabetic rats that received vehicle only (STZ + vehicle). Representative immunoblots are shown in the upper portion, and the relative expression levels of GLUT4 are shown in the lower column (mean \pm SE) obtained from groups of six samples each. $* P<0.05$ compared with $\mathrm{STZ}+$ vehicle.

Abbreviations: GLUT, glucose transporter; GW, GW0742; GW0742, selective PPAR- $\delta$ agonist; PPAR, peroxisome proliferator-activated receptor; SE, standard error; STZ, streptozotocin. 


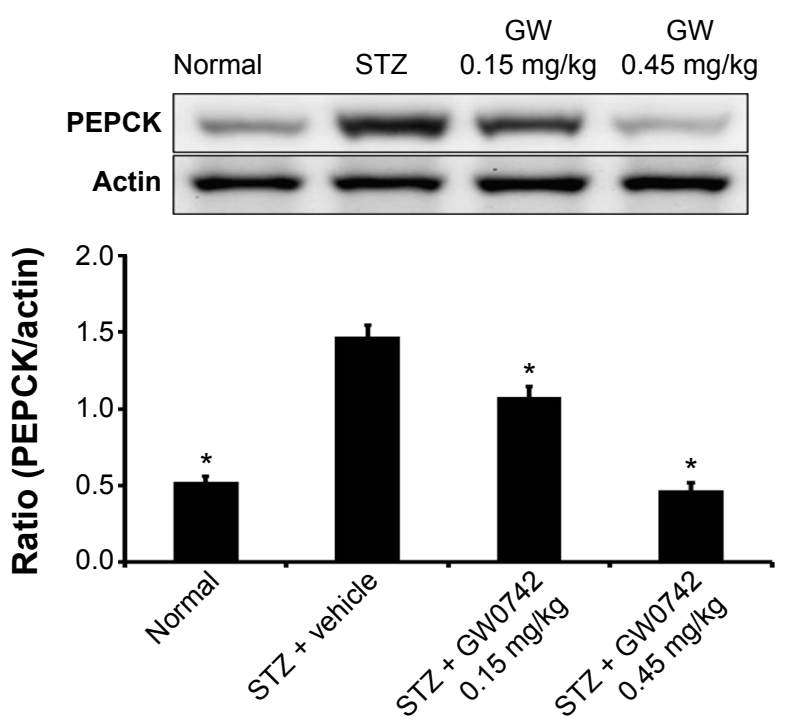

Figure 6 Changes in PEPCK expression induced by GW0742 in the liver of type I-like diabetic rats.

Notes: Changes of PEPCK expression in STZ-induced diabetic rats are shown as STZ for comparison with the changes in normal rats, Normal. Additionally, the effects of GW0742 on diabetic rats are shown as STZ + GW0742 at the indicated dose compared to diabetic rats that received vehicle only (STZ + vehicle). Representative immunoblots are shown in the upper portion, and the relative expression levels of PEPCK are shown in the lower column (mean $\pm \mathrm{SE}$ ) obtained from groups of six samples each. $* P<0.05$ compared with STZ + vehicle.

Abbreviations: GLUT, glucose transporter; GW, GW0742; GW0742, selective PPAR- $\delta$ agonist; PEPCK, phosphoenolpyruvate carboxykinase; PPAR, peroxisome proliferator-activated receptor; SE, standard error; STZ, streptozotocin.

used in animal studies. ${ }^{22}$ GW0742 improved the decreased GIR in a dose-related manner, and this result verified that GW0742 improves IR in vivo. Moreover, insulin sensitivity in diabetic rats was also enhanced by GW0742 in a dose-related manner. Taken together, these data suggest that GW0742 increases insulin sensitivity to improve IR in diabetic rats fed fructoserich chow. These new findings are consistent with a recent report showing that GW0742 ameliorates IR in mice fed highfructose corn syrup. ${ }^{12}$ Therefore, PPAR- $\delta$ shall be considered a new target for the development of drugs to treat IR.

Increase in insulin sensitivity is mainly induced by the enhancement of insulin signals. ${ }^{23}$ PPAR- $\delta$ belongs to the nuclear receptor family, ${ }^{7}$ but the integration of nuclear receptors with insulin signals is still unclear. Details of this enhancement at the cellular level will be investigated in the future. However, hyperglycemia can be reduced by means other than increasing insulin sensitivity. Agent(s) unrelated to insulin can lower blood glucose, which is an insulinotropic action; incretin ${ }^{24}$ and some herbal products ${ }^{25,26}$ may have this ability. Therefore, it will be important to determine whether GW0742 possesses insulinotropic action.

In the type 1-like diabetic rats, bolus injection of GW0742 decreased hyperglycemia in a dose-dependent manner. Mediation of endogenous insulin is negligible in this animal model ${ }^{18}$ because pancreatic cells are mostly damaged by STZ at the treatment dose, thereby suggesting an insulinotropic action of GW0742. Additionally, this action of GW0742 was also blocked by GSK0660 at the dose sufficient to block PPAR- $\delta{ }^{21}$ Thus, activation of PPAR- $\delta$ by GW0742 to produce its insulinotropic action is plausible. However, it has been documented that oral intake of GW0742 did not modify the blood glucose level either in diabetic rats ${ }^{27}$ or in diabetic mice. ${ }^{28}$ This difference seems related to the pharmacokinetic factors because GW0742 is effective through intravenous injection in the present study. Therefore, the pharmacokinetic parameters shall be concerned as GW0742 is going to be applied for oral administration. Moreover, the decreased expression of GLUT4 was reversed by GW0742 in skeletal muscle isolated from the type 1-like diabetic rats. A decrease in insulin-mediated glucose uptake caused by lower expression levels of GLUT4 has been documented in the skeletal muscle, a major site for glucose disposal, of diabetic animal models. ${ }^{14}$ Additionally, the improvement of glucose homeostasis by telmisartan, an angiotensin receptor blocker, has been characterized through selective PPAR- $\delta$ activation in skeletal muscle using knockout mice. ${ }^{29}$ Therefore, PPAR- $\delta$ activation in skeletal muscle is important for the reduction of hyperglycemia, especially the reversion of reduced GLUT4 expression, which promotes glucose uptake. ${ }^{26}$ Importantly, this new finding has not been previously reported. However, it has been documented that GW0742 failed to modify the mRNA level of cultured human myotubes. ${ }^{30}$ Therefore, the effect of GW0742 on basal GLUT4 expression in skeletal muscle shall be investigated in the future.

Hyperglycemia is a consequence of increased hepatic glucose output in concert with reduced peripheral glucose utilization in diabetes, and PEPCK is one of the key enzymes involved in the regulation of hepatic gluconeogenesis. ${ }^{13}$ Moreover, insulin deficiency is clearly associated with a change in hepatic metabolism. ${ }^{13}$ In the present study, we identified increased PEPCK expression in the liver isolated from type 1-like diabetic rats. Treatment with GW0742 attenuated the higher expression of hepatic PEPCK in a dose-related manner. It has been documented that PPAR- $\delta$ functions as a nuclear sensor of dietary fats, capable of modulating immune response through regulation of metabolic programs in liver. ${ }^{31}$ However, modification of PEPCK protein levels by PPAR- $\delta$ has not been previously demonstrated. Therefore, this study shows that GW0742 has the ability to influence hepatic gluconeogenesis through reduction of PEPCK protein levels. This result is consistent with a previous report in mice using the identification of mRNA levels of PEPCK. ${ }^{32}$ Although the mechanism for this inhibition is unknown and 
will be investigated in the future, this study indicated that the hepatic action of GW0742 is related to the production of insulinotropic action in animals.

\section{Conclusion}

In conclusion, the data obtained suggest that GW0742 has the ability to improve IR, in addition to the insulinotropic action in diabetic rats through activation of PPAR- $\delta$. Therefore, PPAR- $\delta$ is a good target for the development of $\operatorname{drug}(\mathrm{s})$ to treat diabetic disorders.

\section{Acknowledgments}

We thank YC Chen for assistance in experiments and Professor YC Tong for editing. The present study was supported in part by a grant from the Tzu Chi College of Technology (TCCT-1022A07), Hualien City, Taiwan.

\section{Disclosure}

The authors report no conflicts of interests in this work.

\section{References}

1. Akkati S, Sam KG, Tungha G. Emergence of promising therapies in diabetes mellitus. J Clin Pharmacol. 2011;51(6):796-804.

2. Shaw JE, Sicree RA, Zimmet PZ. Global estimates of the prevalence of diabetes for 2010 and 2030. Diabetes Res Clin Pract. 2010;87(1):4-14.

3. Fonseca VA, Kirkman MS, Darsow T, Ratner RE. The American Diabetes Association diabetes research perspective. Diabetes. 2012;61(6):1338-1345.

4. Badin PM, Vila IK, Louche K, et al. High-fat diet-mediated lipotoxicity and insulin resistance is related to impaired lipase expression in mouse skeletal muscle. Endocrinology. 2013;154(4):1444-1453.

5. Chiu YJ, Chung HH, Yeh CH, Cheng JT, Lo SH. Improvement of insulin resistance by Chlorella in fructose-rich chow-fed rats. Phytother Res. 2011;25(9):1306-1312.

6. Kliewer SA, Lehmann JM, Milburn MV, Willson TM. The PPARs and PXRs: nuclear xenobiotic receptors that define novel hormone signaling pathways. Recent Prog Horm Res. 1999;54:345-367.

7. Feige JN, Gelman L, Michalik L, Desvergne B, Wahli W. From molecular action to physiological outputs: peroxisome proliferator-activated receptors are nuclear receptors at the crossroads of key cellular functions. Prog Lipid Res. 2006;45(2):120-159.

8. Barish GD, Narkar VA, Evans RM. PPAR $\delta$ : a dagger in the heart of the metabolic syndrome. J Clin Invest. 2006;116(3):590-597.

9. Benetti E, Patel NS, Collino M. The role of PPAR $\beta / \delta$ in the management of metabolic syndrome and its associated cardiovascular complications. Endocr Metab Immune Disord Drug Targets. 2011;11(4):273-284.

10. Michalik L, Auwerx J, Berger JP, et al. International Union of Pharmacology. LXI. Peroxisome proliferator-activated receptors. Pharmacol Rev. 2006;58(4):726-741.

11. Li L, Luo Z, Yu H, et al. Telmisartan improves insulin resistance of skeletal muscle through peroxisome proliferator-activated receptor- $\delta$ activation. Diabetes. 2013;62(3):762-774.

12. Benetti E, Mastrocola R, Rogazzo M, et al. High sugar intake and development of skeletal muscle insulin resistance and inflammation in mice: a protective role for PPAR- $\delta$ agonism. Mediators Inflamm. 2013;2013:509502.

13. Consoli A, Nurjhan N, Capani F, Gerich J. Predominant role of gluconeogenesis in increased hepatic glucose production in NIDDM. Diabetes. 1989;38(5):550-557.
14. Berger J, Biswas C, Vicario PP, Strout HV, Saperstein R, Pilch PF. Decreased expression of the insulin-responsive glucose transporter in diabetes and fasting. Nature. 1989;340(6228):70-72.

15. Cheng MF, Chen LJ, Cheng JT. Decrease of Klotho in the kidney of streptozotocin-induced diabetic rats. J Biomed Biotechnol. 2010; 2010:513853.

16. Wu HT, Chen CT, Cheng KC, Li YX, Yeh CH, Cheng JT. Pharmacological activation of peroxisome proliferator-activated receptor delta improves insulin resistance and hepatic steatosis in high fat diet-induced diabetic mice. Horm Metab Res. 2011;43(9):631-635.

17. Finegood DT, Bergman RN, Vranic M. Estimation of endogenous glucose production during hyperinsulinemic-euglycemic glucose clamps. Comparison of unlabeled and labeled exogenous glucose infusates. Diabetes. 1987;36(8):914-924.

18. Cheng JT, Huang CC, Liu IM, Tzeng TF, Chang CJ. Novel mechanism for plasma glucose-lowering action of metformin in streptozotocininduced diabetic rats. Diabetes. 2006;55(3):819-825.

19. Haskova Z, Hoang B, Luo G, et al. Modulation of LPS-induced pulmonary neutrophil infiltration and cytokine production by the selective PPARß/8 ligand GW0742. Inflamm Res. 2008;57(7):314-321.

20. Kuo SC, Ku PM, Chen LJ, Niu HS, Cheng JT. Activation of receptors $\delta$ (PPAR $\delta$ ) by agonist (GW0742) may enhance lipid metabolism in heart both in vivo and in vitro. Horm Metab Res. 2013;45(12):880-886.

21. Chen ZC, Lee KS, Chen LJ, Wang LY, Niu HS, Cheng JT. Cardiac peroxisome proliferator-activated receptor $\delta$ (PPAR $\delta$ ) as a new target for increased contractility without altering heart rate. PLoS One. 2013;8(5):e64229.

22. Yang G, Li CL, Tian H, et al. Different establishing conditions of hyperinsulinemic-euglycemic clamp technique among different groups. Zhonghua Yi Xue Za Zhi. 2012;92(34):2410-2414.

23. Samuel VT, Shulman GI. Mechanisms for insulin resistance: common threads and missing links. Cell. 2012;148(5):852-871.

24. Ramesh N, Mortazavi S, Unniappan S. Nesfatin-1 stimulates glucagonlike peptide-1 and glucose-dependent insulinotropic polypeptide secretion from STC-1 cells in vitro. Biochem Biophys Res Commun. 2015;462(2):124-130.

25. Puri D. The insulinotropic activity of a Nepalese medicinal plant Biophytum sensitivum: preliminary experimental study. J Ethnopharmacol. 2001;78(1):89-93.

26. Maroo J, Vasu VT, Aalinkeel R, Gupta S. Glucose lowering effect of aqueous extract of Enicostemma littorale Blume in diabetes: a possible mechanism of action. J Ethnopharmacol. 2002;81(3):317-320.

27. Quintela AM, Jiménez R, Gómez-Guzmán M, et al. Activation of peroxisome proliferator-activated receptor- $\beta /-\delta(\operatorname{PPAR} \beta / \delta)$ prevents endothelial dysfunction in type 1 diabetic rats. Free Radic Biol Med. 2012;53(4):730-741.

28. Matsushita Y, Ogawa D, Wada J, et al. Activation of peroxisome proliferator-activated receptor delta inhibits streptozotocin-induced diabetic nephropathy through anti-inflammatory mechanisms in mice. Diabetes. 2011;60(3):960-968.

29. Perez-Schindler J, Svensson K, Vargas-Fernandez E, Santos G, Wahli W, Handschin C. The coactivator PGC-1alpha regulates skeletal muscle oxidative metabolism independently of the nuclear receptor PPARbeta/ delta in sedentary mice fed a regular chow diet. Diabetologia. 2014;57(11):2405-2412.

30. Krämer DK, Al-Khalili L, Perrini S, et al. Direct activation of glucose transport in primary human myotubes after activation of peroxisome proliferator-activated receptor delta. Diabetes. 2005;54(4):1157-1163.

31. Liu S, Hatano B, Zhao M, et al. Role of peroxisome proliferatoractivated receptor $\beta / \delta$ in hepatic metabolic regulation. $J$ Biol Chem. 2011;286(2):1237-1247.

32. Chen W, Wang LL, Liu HY, Long L, Li S. Peroxisome proliferatoractivated receptor delta-agonist, GW501516, ameliorates insulin resistance, improves dyslipidaemia in monosodium L-glutamate metabolic syndrome mice. Basic Clin Pharmacol Toxicol. 2008;103(3): 240-246. 


\section{Publish your work in this journal}

Drug Design, Development and Therapy is an international, peerreviewed open-access journal that spans the spectrum of drug design and development through to clinical applications. Clinical outcomes, patient safety, and programs for the development and effective, safe, and sustained use of medicines are a feature of the journal, which

has also been accepted for indexing on PubMed Central. The manuscript management system is completely online and includes a very quick and fair peer-review system, which is all easy to use. Visit http://www.dovepress.com/testimonials.php to read real quotes from published authors.

Submit your manuscript here: http://www.dovepress.com/drug-design-development-and-therapy-journal 